\title{
A Note on Dynamic Programming in Accounts Receivable Management*
}

\author{
Y. M. I. Dirickx ${ }^{1}$ and K.-P. Kistner ${ }^{2}$ \\ 1 Twente University of Technology, Department of Applied Mathematics, NL-7500 AE Enschede, The Netherlands \\ 2 Fakultät für Wirtschaftswissenschaften, Universität Bielefeld, D-4800 Bielefeld
}

Received October 15, 1980 / Accepted in revised form October 14, 1981

Summary. The paper considers a dynamic programming formulation of the accounts receivable problem for single outstanding amounts. An optimal collection policy can be computed efficiently by invoking a "planning horizon" result that determines a time period beyond which the decision process cannot extend. The optimality of socalled monotone policies is shown under rather intuitive restrictions on the collection probabilities.

Zusammenfassung. Das Problem der Kredituiberwachung wird als einfaches dynamisches Programm formuliert. Unter Ausnutzung endlicher oberer Schranken für den Planungshorizont, die einen Zeitpunkt determinieren, über den hinaus der Entscheidungsprozeß nicht ausgedehnt werden muß, können einfache Strategien für die zeitliche Koordination von Mahnaktionen hergeleitet werden. Es wird gezeigt, daß unter recht realitätsnahen Annahmen über die Rückzahlungswahrscheinlichkeiten sogenannte monotone Politiken optimal sind.

\section{Introduction}

The growing expansion of consumer credit (e.g., credit cards) resulted in various investigations dealing with various aspects of establishing optimal credit control policies. Roughly speaking, this literature can be divided in two areas: those studies dealing with the decision to grant credit or not and with the accounts receivable management (with as its extreme the study of bad debt manage-

* This research was initiated during Klaus-Peter Kistner's stay at the European Institute for Advanced Studies in Management, Brussels, and completed during Yvo Dirickx's visit at Bielefeld University ment). The credit granting decision is basically a decision problem under uncertainty with costly information; typical approaches are based on discriminant analysis (see Forgy and Meyers [5]) or on Bayesian analysis (see Bierman and Hausman [1], Dirickx and Wakeman [4]), for a review the reader may consult Wakeman [8]. Accounts receivable management, on the other hand, deals with the determination of an optimal collection policy for late payments once credit has been granted, i.e., the management of "doubtful accounts" (including the socalled bad debts).

In fact, one of the first applications of Markov chain analysis, described in Cyert et al. [3] dealt with the estimation of doubtful accounts based on outstanding balances, using various (by now, well-known) limiting results of Markov chain theory. Recently, Corcoran [2], incorporated exponential smoothing into the estimation procedure of a Cyert - Davidson - Thompson type model. Liebman [7] utilized a similar framework, imposed a cost structure on it, and formulated a Markov decision problem to determine an optimal follow-up policy (excluding, however, an explicit treatment of bad debts). Liebman's model is based on aggregated data describing transition probabilities between various age classes for varying account size (charge volumes).

Liebman showed that an optimal policy may have the "pathological" property in being non-monotonic, i.e., that more drastic (expensive) collection actions may be taken before less drastic (cheaper) actions (see Section 2 below for a discussion of "monotone" policies). This is due to the fact that the balance of the accounts but not the single outstanding amount is considered. Hence, an account may be classified as "bad" if a recent relatively high claim will be added to an old but small claim.

In contrast, the present study develops a model based on the notion of deriving an optimal follow-up policy for single outstanding amounts; it will be shown 
that, under a certain condition, "pathologies" do not arise. Bad debt management is not explicitly treated.

The model is specified in the next section. A result on the optimality of monotone policies is described in Section 3. A useful "planning horizon" result is derived in Section 4.

\section{The Model}

Consider a $n$-stage decision problem where the stages are indexed by $i, l \leqslant i \leqslant n$. Let $x$ be the amount of credit outstanding at the beginning of the decision process, and assume that this amount is either paid back entirely at a particular stage or not (but possibly in a later stage). Then, denoting by $x_{i}$ the amount outstanding at the beginning of stage $i$,

$x_{i}= \begin{cases}x>0 & \text { if no collection occurred in stage } 1 \text { to } i-1, \\ 0 & \text { if collection occurred before stage } i .\end{cases}$

In each stage a finite set of actions $A=\{0,1, \ldots, m-1$, $m$ \} is available to the decision maker to "influence" the pay-back behavior. With each of these actions a cost $c_{k}$, $k \in A$, is associated. Action 0 corresponds to do nothing, actions 1 to $m-1$ correspond to increasingly costly, but also increasingly effective actions. For instance " 1 " could be to write a letter, " 2 " to send a special delivery letter, " 3 " to send a cable, etc. Action " $m$ ", the write-off action, implies that either the credit is payed back immediately or that the customer is taken out of the routine follow-up procedure, that no more credit will be granted and that the "bad debt" is transmitted to the bad debt collection department. In this case, an amount of $u(x)<x$ is credited, reflecting the discounted expected value of proceeds from future collecting efforts.

We assume that

$0=c_{0}<c_{1}<\ldots<c_{m-1}$.

Let $q_{i k}=q_{i k}(x)(k=1, \ldots, m)$ denote the probability of collecting the outstanding amount $x$ in stage $i$ when action $k$ is taken. We assume that $q_{i 0}<q_{i 1}<\ldots<q_{i m-1}$ for all $i=1, \ldots, n-1$, reflecting the increasing effectiveness or more expensive actions. Furthermore a credit of age $n$ is automatically written off.

Define $f_{i}(x)$ as the maximal expected discounted profit for stages $i$ through $n$ when the outstanding amount is equal to $x$, then and

$f_{i}(0) \equiv 0 \quad i=1, \ldots, n$

where $\alpha \in(0,1]$ denotes the discount factor.

\section{The Structure of Optimal Policies}

Before proceeding to characterize the structure of optimal solution to (1), we make here a simple observation:

$f_{i}(x) \leqslant x \quad i=1, \ldots, n$

Although (1) is easy to solve, we will show that, under certain conditions, further simplifications come about. In particular, we will establish a sufficient condition guaranteeing the optimality of monotone policies.

\section{Definition 1}

A policy is a sequence of actions $\delta(i, x) \in A(i=1, \ldots, n)$. A policy is said to be a monotone policy if

$m \geqslant \delta(i+1, x) \geqslant \delta(i, x) \quad$ for $\quad i=1, \ldots, n-1$;

$\delta(n, x)=m \quad$ and $\quad \delta(i, 0) \equiv 0$.

A monotone policy corresponds to the intuitive notion that a more expensive action should never preceded a less expensive one. Note that monotonicity is taken in a weak sense. Inspection reveals that monotone policies need not be optimal under the assumptions introduced up to this point.

\section{Definition 2}

An action $k \in A$ dominates an action $l \in A(k, l \neq m)$ in stage $i$ and write $k>l$ iff

$h_{i k}(x)>h_{i l}(x)$

where

$h_{i j}(x)=q_{i j} x-c_{j}+\alpha\left(1-q_{i j}\right) f_{i+1}(x) \quad j=k, l$.

To establish a sufficient condition we will utilize the following

$f_{i}(x)=\max \left\{\begin{array}{l}\max _{0 \leqslant k \leqslant m-1}\left\{q_{i k} x-c_{k}+\alpha\left(1-q_{i k}\right) f_{i+1}(x)\right\} \\ q_{i m^{x}} x-c_{m}+\alpha\left(1-q_{i m}\right) u(x)\end{array}\right\} i=1, \ldots, n-1$

$f_{n}(x)=q_{n m} x-c_{m}+\alpha\left(1-q_{n m}\right) u(x)$ 


\section{Lemma 1}

If $q_{i-1, k} \geqslant q_{i k}$ for all $i$ and $k$, then $f_{i-1}(x) \geqslant f_{i}(x)$ for $i=2, \ldots, n$.

\section{Proof}

The proof is by induction.

Clearly,

$$
\begin{aligned}
f_{n-1}(x) & \geqslant q_{n-1 m}(x-\alpha u(x))-c_{m}+\alpha u(x) \\
& \geqslant q_{n m}(x-\alpha u(x))-c_{m}+\alpha u(x) \\
& =f_{n}(x),
\end{aligned}
$$

establishing the result for $i=n-1$.

For an arbitrary $i$,

$$
\begin{aligned}
& f_{i-1}(x)= \max \left\{\max _{0 \leqslant k \leqslant m-1}\left\{q_{\mathrm{i}-1, k}\left(x-\alpha f_{i}(x)\right)-c_{k}+\alpha f_{i}(x)\right\} ;\right. \\
& \geqslant\left.q_{i-1, m}(x-\alpha u(x))-c_{m}+\alpha u(x)\right\} \\
& \max \left\{\max _{0 \leqslant k \leqslant m-1}\left\{q_{i k} x-c_{k}+\alpha\left(1-q_{i k}\right) f_{i+1}(x)\right\} ;\right. \\
&\left.\quad q_{i m}(x-\alpha u(x))-c_{m}+\alpha u(x)\right\} \\
&=f_{i}(x) .
\end{aligned}
$$

This holds by using the assumption of the Lemma 1 and the inductive hypothesis, and since $x-\alpha f_{i}(x) \geqslant 0$.

New we can prove

\section{Theorem 1}

If for all $k, l \in A$

(i) $c_{k}>c_{l} \Rightarrow q_{i k}>q_{i l}$ for all $i=1,2, \ldots, n$

(ii) $q_{i-1, k} \geqslant q_{i k}$ for $i=2, \ldots, n$

(iii) $q_{i k}>q_{i l} \Rightarrow q_{i-1, k}-q_{i k} \leqslant q_{i-1, l}-q_{i l}$, for all $i=2, \ldots, n$

\section{then a monotone policy is optimal.}

ad (i) This reflects efficiency considerations: a possible action will not be considered, if it is more expensive, but less effective than another action,

ad (ii) it implies that the effects of an action wears off with the age of a credit, and

ad (iii) this states that a more expensive action wears off slower than a less expensive action.

\section{Proof (by contradiction)}

Let $l<k<m \in A$. Consider any given policy with the following properties: (a) $l \stackrel{i}{\succ} k$

(b) $k \stackrel{i-1}{>} l$

for any $i \leqslant n$. From Definition 2 and (i) we obtain

$\left(q_{i k}-q_{i l}\right)\left(x-\alpha f_{i+1}(x)\right)<c_{k}-c_{l}$

and

$\left(q_{i-1, k}-q_{i-1, l}\right)\left(x-\alpha f_{i}(x)\right)>c_{k}-c_{l}$

or, using (iii), we derive from (3a)-(3b)

$\left(q_{i k}-q_{i l}\right)\left(x-\alpha f_{i+1}(x)\right)<c_{k}-c_{l}$

$<\left(q_{i-1, k}-q_{i-1, l}\right)\left(x-\alpha f_{i}(x)\right)$

$\leqslant\left(q_{i k}-q_{i l}\right)\left(x-\alpha f_{i}(x)\right)$

Hence, $f_{i+1}(x)>f_{i}(x)$, a contradiction to Lemma 1.

Thus, a policy with properties (a) and (b) cannot be optimal.

Theorem 1 reflects well established customs in accounts receivable management: collecting efforts are started with "soft" (that is less expensive) actions followed by "tough" (that is more expensive) actions, if the former do not result in repayment. Furthermore, if the conditions of Theorem 1 are met, the dynamic programming recursion (1) is greatly facilitated: If action $k \leqslant m$ is taken in $t$, only actions $l \leqslant k$ have to be considered in $i<\mathrm{t}$. Hence, computing times as well as storage requirements are cut down.

\section{The Choice of a Planning Horizon}

To solve the dynamic program (1) recursively, a finite planning horizon $n$ has to be specified, so that collecting efforts after period $n$ have not to be considered. In some cases such a planning horizon is suggested by data available: if no positive probabilities can be attached to the collection or if the cost of all collection actions exceeds its expected outcome for outstanding amounts older than $n$ periods, possible actions beyond this horizon are irrelevant for the optimal policy. In other cases it will be possible to determine a finite planning horizon, using monotonicity properties.

For this end we introduce a fictitious policy $\mu_{n}$ defined for arbitrary, but sufficiently large horizon $n$,

$$
\begin{aligned}
& \mu_{n}(i, 0)=0 \\
& \mu_{n}(i, x)= \begin{cases}m-1 & i=1,2, \ldots, n \\
m & i=1,2, \ldots, n-1\end{cases}
\end{aligned}
$$


which can be implemented costlessly, in the sense that $c_{m-1}=0$.

In other words, we use the most effective actions without considering cost of collecting efforts.

Now, it pays to write off the debt in a particular period $t$ rather than in period $t+1$, if

$$
\begin{aligned}
& \Delta_{t}=-c_{m}+q_{t m} x+\alpha\left(1-q_{t m}\right) u(x) \\
& -q_{t, m-1} x-\alpha\left(1-q_{t, m-1}\right)\left[-c_{m}+q_{t+1, m} x\right. \\
& \left.+\alpha\left(1-q_{t+1, m}\right) u(x)\right] \geqslant 0
\end{aligned}
$$

To avoid that (4) has no solution in $t$, it suffices to assume that $\alpha u(x) \geqslant c_{m}$ and that $q_{i k} \rightarrow 0$ as $i \rightarrow \infty$ which is, of course, very plausible.

If it pays to write off the debt in period $t$ rather than in period $t+1$, even if a free collecting action, more effective than any other action, is available, it will certainly pay to write off in period $t$ when costs are taken into account.

However, condition (4) does not exclude that it pays to postpone the write-off action for more than one period. To rule out this atypical behavior, we have to show that $\Delta_{i}$ does not decrease of $i>t$. It is easy to check that $\Delta_{i+1}-\Delta_{i} \geqslant 0$, iff

$$
\begin{aligned}
& {\left[\left(q_{i m-1}-q_{i+1, m-1}\right)-\left(q_{i m}-q_{i+1, m}\right)\right] x} \\
& -\alpha u(x)\left[\alpha\left(q_{i, m-1}-q_{i+1, m-1}\right)-\left(q_{i, m}-q_{i+1, m}\right)\right] \\
& +\left(q_{i, m-1}-q_{i+1, m-1}\right) c_{m} \geqslant \alpha\left[q_{i+2, m}\left(1-q_{i+1, m-1}\right)\right. \\
& \left.-q_{i+1, m}\left(1-q_{i, m-1}\right)\right][x-\alpha u(x)]
\end{aligned}
$$

As $x>\alpha u(x)$, it is sufficient for (5) to hold that for all $i \geqslant t$

(i) $q_{i m}\left(1-q_{i-1, m-1}\right) \leqslant q_{i-1, m}\left(1-q_{i-2, m}\right)$

(ii) $q_{i k} \leqslant q_{i-1, k}$ for $k=m-1, m$

$$
q_{i m} \geqslant q_{i, m-1}
$$

(iii) $q_{i, m-1}-q_{i+1, m-1} \geqslant q_{i m}-q_{i+1, m}$

Here, (ii) and (iii) are extensions of the corresponding conditions of Theorem 1. As $q_{i k}$ is small for large $i$, the mixed expressions $q_{i m} \cdot q_{i-1, m-1}$ will tend to zero faster than $q_{i m}$; hence (i) will hold for large $i$. Furthermore, when (i) is valid for $t$ it is valid for $i>t$, if

$$
\begin{aligned}
& \text { (iv) } q_{i m}-q_{i+1, m} \leqslant q_{i-1, m}-q_{i m} \\
& q_{i, m-1}-q_{i+1, m-1} \leqslant q_{i-1, m-1}-q_{i, m-1}
\end{aligned}
$$

For example, condition (iv) is fulfilled, if the collection probabilities $q_{i k}(k=m-1, m)$ are bounded by a geometrical series.

Now we show that $n_{,}^{*}=t$ is a finite planning horizon for the policy $\mu_{n}$. If it is optimal to write off the debt in period $t, x_{i}=0$ and $f_{i}\left(x_{i}\right)=0$ for $i>t$. Hence neither an increase nor a reduction of the planning horizon $n$ to $t$ will affect the maximal discounted profits for the stages $i$ through $t$ or the optimal policy.

If it does not pay to use the most effective action $m-1$ without considering cost, it will not pay all the more to use a less effective, costly action. Consequently, $t$ is an upper bound for the planning horizon for the original problem.

Hence, the following procedure to determine an upper bound for the planning horizon can be used

(a) Find the smallest $t$ for which (4) holds. If (iii) holds for all $i \geqslant t$, start the recursion (1) with $n=t$.

(b) If (iii) does not hold for $i=t$, find the smallest $\tau$ so that (iii) holds for all $i \geqslant \tau$. If (iv) holds, (iii) holds for all $i \geqslant \tau$.

Start the recursion (1) with $n=\tau$.

Summarizing, it should be stressed that account receivable management can be mapped by a simple dynamic programming model which can be solved by using standard methods. Furthermore, if some monotonicity conditions on the collection probabilities are introduced, the optimization is even more straightforward and "pathologies" of the type encountered in Liebman [6] do not arise. A suitable upper bound for the planning horizon can be found, so that (1) can be solved recursively.

\section{References}

1. Bierman H, Hausman WH (1970) The credit granting decision. Manag Sei 16:B519-532

2. Corcoran AW (1978) The use of exponentially-smoothed transition matrices to improve forecasting of cash flows from accounts receivable. Manag Sci 24:732-739

3. Cyert R, Davidson H, Thompson SL (1962) Estimation of the allowance for doubtful accounts by Markov chains. Manag Sci 8:287-303

4. Dirickx YMI, Wakeman L (1976) An extension of the Bierman-Hausman model for credit granting. Manag Sci 22:12291237

5. Forgy EW, Meyers JH (1963) The development of numerical credit evaluation systems. J Am Stat 58:799-806

6. Kistner K-P, Dirickx YMI (1980) Monotone Strategien zur Kontrolle von Außenständen. In: Hauptmann H, Schenk K-E (Hrsg) Anwendungen der Systemtheone und Kybernetik in Wirtschaft und Verwaltung. Duncker \& Humblot, Berlin, S 64-79

7. Liebman LH (1972) A Markov decision model for selecting optimal credit control policies. Manag Sci 18:519-525

8. Wakeman L (1974) Bayesian methods in credit management, unpublished Ph.D.-Dissertation, M.I.T. 